\title{
COVID-19 and Myositis: What We Know So Far
}

\author{
Ahmad Saud $^{1}$ (D) $\cdot$ R Naveen $^{2}$ (D) $\cdot$ Rohit Aggarwal $^{3}$ (D) Latika Gupta $^{2}$ (D) \\ Accepted: 22 May 2021 / Published online: 3 July 2021 \\ (C) The Author(s), under exclusive licence to Springer Science+Business Media, LLC, part of Springer Nature 2021
}

\begin{abstract}
Purpose Myositis as a rare manifestation of COVID-19 is only recently being reported. This review examines the current literature on COVID-19-induced myositis focusing on etiopathogenesis, clinical presentations, diagnostic practices, and therapeutic challenges with immunosuppression, and the difficulties experienced by rheumatologists in established myositis in the COVID-19 era.

Recent Findings COVID-19 is associated with a viral myositis attributable to direct myocyte invasion or induction of autoimmunity. COVID-19-induced myositis may be varied in presentation, from typical dermatomyositis to rhabdomyolysis, and a paraspinal affliction with back pain. It may or may not present with acute exponential elevations of enzyme markers such as creatine kinase (CK). Virus-mediated muscle inflammation is attributed to ACE2 (angiotensin-converting enzyme) receptormediated direct entry and affliction of muscle fibers, leading on to innate and adaptive immune activation. A greater recognition of the stark similarity between anti-MDA5-positive myositis with COVID-19 has thrown researchers into the alley of exploration — finding common etiopathogenic basis as well as therapeutic strategies. For patients with established myositis, chronic care was disrupted during the pandemic with several logistic challenges and treatment dilemmas leading to high flare rates. Teleconsultation bridged the gap while ushering in an era of patient-led care with the digital transition to tools of remote disease assessment.

Summary COVID-19 has brought along greater insight into unique manifestations of COVID-19-related myositis, ranging from direct virus-induced muscle disease to triggered autoimmunity and other etiopathogenic links to explore. A remarkable shift in the means of delivering chronic care has led patients and caregivers worldwide to embrace a virtual shift with teleconsultation and opened doorways to a new era of patient-led care.
\end{abstract}

Keywords COVID-19 $\cdot$ Myositis · Idiopathic inflammatory myopathy $\cdot$ Rhabdomyolysis $\cdot$ Dermatomyositis $\cdot$ Myasthenia Telemedicine $\cdot$ Tele-triage $\cdot$ Immunopathogenesis

This article is part of the Topical Collection on Inflammatory Muscle Disease

Latika Gupta

drlatikagupta@gmail.com

1 Department of Medicine, Royal College of Surgeons Ireland, Dublin, Ireland

2 Department of Clinical Immunology and Rheumatology, C Block, Sanjay Gandhi Postgraduate Institute of Medical Sciences, Raebareilley Road, Lucknow 226014, India

3 Department of Medicine, University of Pittsburgh, Pittsburgh, PA, USA

\section{Introduction}

The ongoing Coronavirus disease 2 pandemic (COVID-19) has brought several interesting observations to the fore, ranging from virus-induced muscle disease to a possibility of virus-triggered inflammation in patients with long-standing chronic autoimmune diseases $[1,2]$. The initial pandemic period left physicians grappling with the uncertainty of the course of COVID-19 in patients with severe and disabling rheumatic diseases, in particular the idiopathic inflammatory myopathies (IIM) [3•]. Moreover, extended lockdowns in various parts of the world hampered mobility and access to care for most patients [4••].

COVID-19 is a multisystem disease that presents with a plethora of manifestations involving the lungs, liver, kidneys, and gastrointestinal tract, among others. Emerging evidence 
suggests that the acute inflammatory response and production of autoantibodies contribute to morbidity observed in COVID-19 [5]. Lasting effects are observed in some individuals at 6 months or even longer after recovery. While fever, cough, and sore throat have been the most reported symptoms associated with the disease, published case reports have recently started to describe more atypical and rarer presentations of infection with Severe Acute Respiratory Syndrome Coronavirus 2 (SARS-CoV-2). It is apparent now that the musculoskeletal system is not spared either [6] with musculoskeletal manifestations of COVID-19 ranging from a mild elevation of creatine kinase (CK) with mild or no weakness to severe rhabdomyolysis.

This review aims to examine current knowledge available on COVID-19-related myositis, including but not limited to the presentation, diagnostic challenges, currently proposed disease mechanisms, and management. Furthermore, we discuss the complexity of administering immunosuppressive treatment in diagnosed cases of IIM, who may be at risk for contracting COVID-19. We also explore the effect of the pandemic on the management of the idiopathic inflammatory myopathies (IIM) and the proposed directions for care of these debilitating disorders in the future.

\section{Muscle Involvement in COVID-19}

Alongside the typical respiratory manifestations like cough, fever, and sore throat, proximal muscle weakness is increasingly being reported as a manifestation causing significant morbidity in occasional COVID-19 patients. Myalgia is reported extensively in the literature as a common musculoskeletal manifestation of COVID-19 infection, presenting in nearly half of all COVID-19-infected patients. COVID-19-related myositis and consequently rhabdomyolysis are other reported manifestations, albeit relatively rare [7, 8]. By July 2020, a single case of COVID-19-related myositis had been reported in the literature [9]. Since then, several case reports and series reporting a virus-induced myositis attributed to COVID-19 disease have been published. The muscle involvement may vary from an asymptomatic elevation of $\mathrm{CK}$ to severe rhabdomyolysis.

\section{Acute Viral Myositis}

Nearly 23 patient cases of myositis attributable to COVID-19 have been described so far. COVID-19-induced myositis may vary in presentation, ranging from frank muscle weakness to typical dermatomyositis replete with classic rashes, or mere back pain with muscle disease on MRI. Most patients test positive for COVID-19 on initial presentation with most reported being males aged 33-87. Noteworthily, COVID-19 may or may not present with acute exponential elevations of enzyme markers such as CK, and muscle enzymes may not necessarily have a direct bearing on prognosis.

\section{Rhabdomyolysis}

Rhabdomyolysis is one of the rare and severe complications of COVID-19 infection which can be an initial presentation in some cases $[10,11]$. In such cases, patients present with typical COVID-19 symptoms such as fever, cough, myalgia, and shortness of breath as well as manifest acute lower limbdominant symmetric muscle weakness and subsequently go on to develop rhabdomyolysis associated with elevated $\mathrm{CK}$ levels. A study reported a peak CK value as high as 33,000 $\mathrm{U} / \mathrm{L}[12 \bullet]$. In this setting, they may present with frank muscle weakness, which is profound, proximal, lower limb-dominant, acute, and symmetric. At times, the patients are critically ill and requiring ventilatory support. The only manifestation of rhabdomyolysis in these may be myoglobinuria (dark urine) and acute kidney injury (AKI) needing hemodialysis with raised $\mathrm{CK}>5000 \mathrm{IU} / \mathrm{L}[13 \bullet, 14 \bullet, 15 \bullet]$. Rhabdomyolysis can be fatal, with casualties reported in nearly $45 \%$ (4 of 9 reported) of those with this presentation over a short follow-up duration (Table 1). Among all reported cases $(n=23)$ of COVID-19-related myositis so far, $21.7 \%(n=5)$ succumbed to the illness of which $80 \%(n=4)$ had rhabdomyolysis.

Therefore, rhabdomyolysis should be suspected in all cases with soaring muscle enzymes with or without AKI in the critical care setting where a formal muscle examination may or may not be feasible. MRI findings of intramuscular hemorrhage, potentially in the proximal muscles like the shoulder, help differentiate these patients from myositis patients in whom edema would be found in the absence of hypointense attenuation [12•]. In general, patients diagnosed with rhabdomyolysis appear to have negative myositis-specific autoantibodies and higher CK levels than those without this severe complication (Table 1) again highlighting the need for close monitoring of CK levels.

\section{Dermatomyositis}

Another manifestation of COVID-19-induced muscle disease is classic dermatomyositis, replete with rashes, muscle weakness, and interstitial lung disease. The latter two can occur in both COVID-19 and de novo myositis, making timely diagnosis challenging. However, the presence of typical rashes such as heliotrope with periorbital edema, malar erythema, or diffuse facial rashes may guide diagnosis of dermatomyositis. Sometimes, less specific erythematous rashes over the extensor surfaces of limbs (knees, elbows) and trunk may be the only clue. Muscle weakness is invariably present, being symmetric, proximal, and involving upper as well as lower limbs. Severe bulbar weakness is also reported [16•]. CK is usually elevated but can occasionally be normal when muscle 


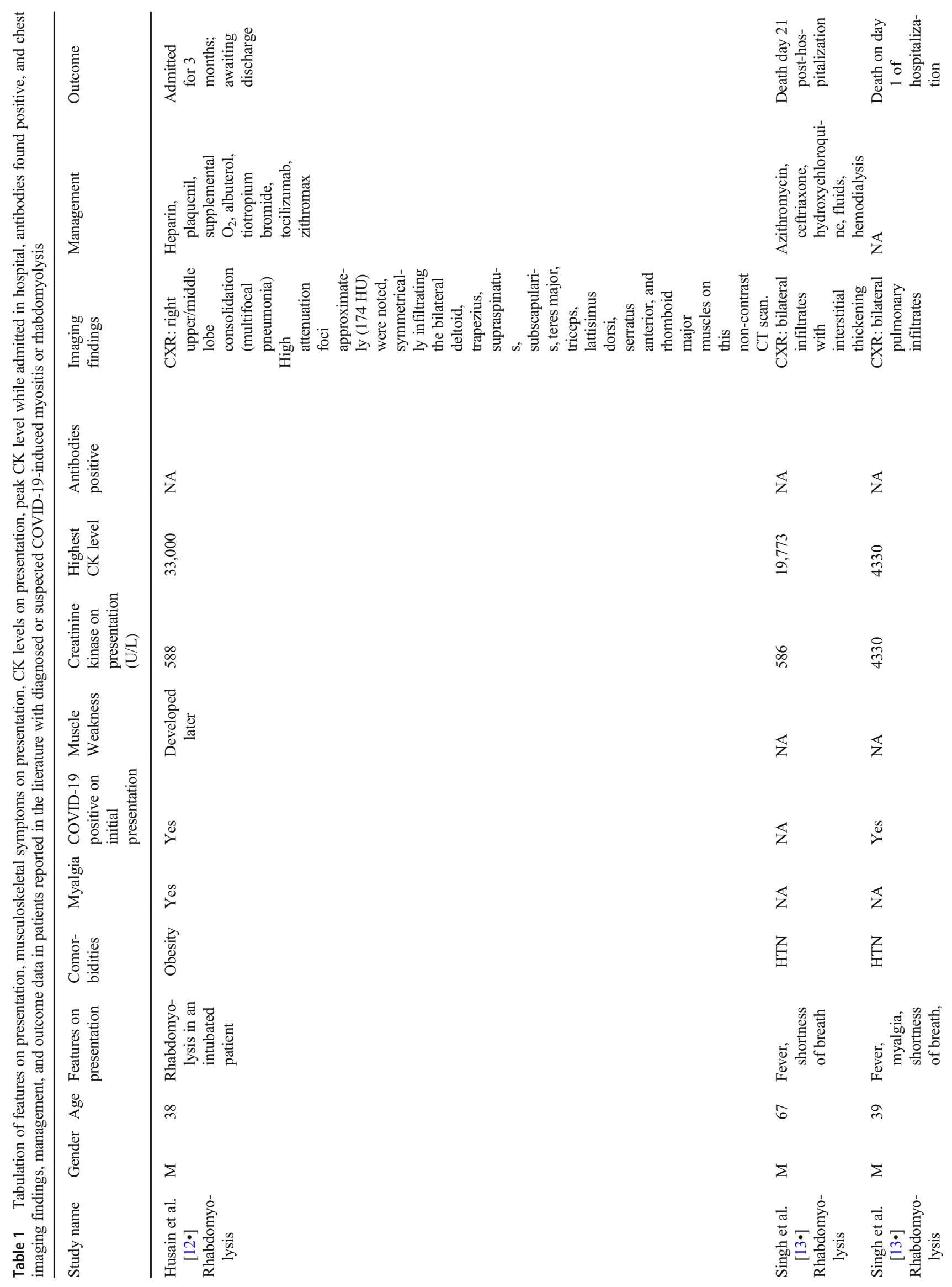




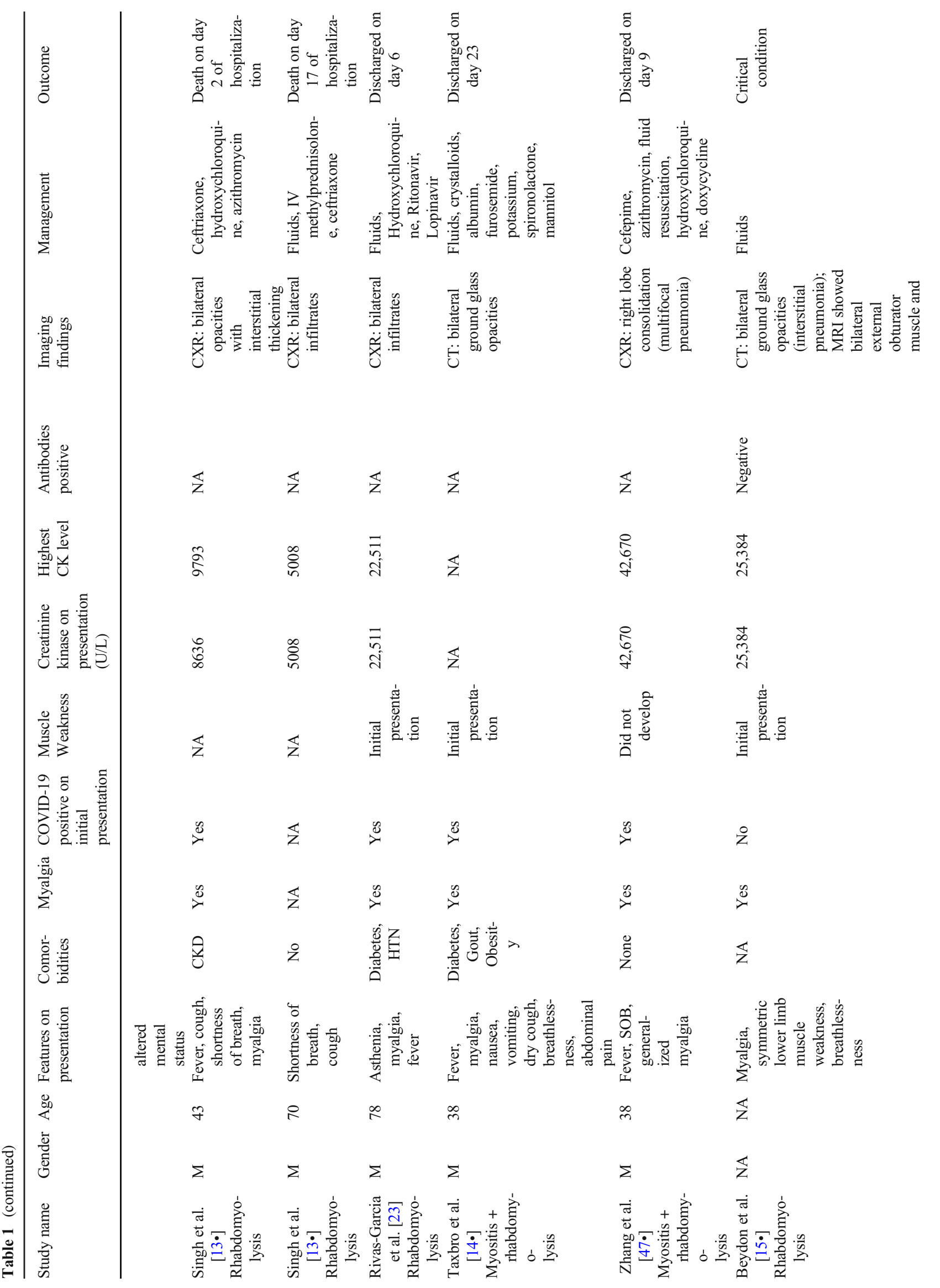




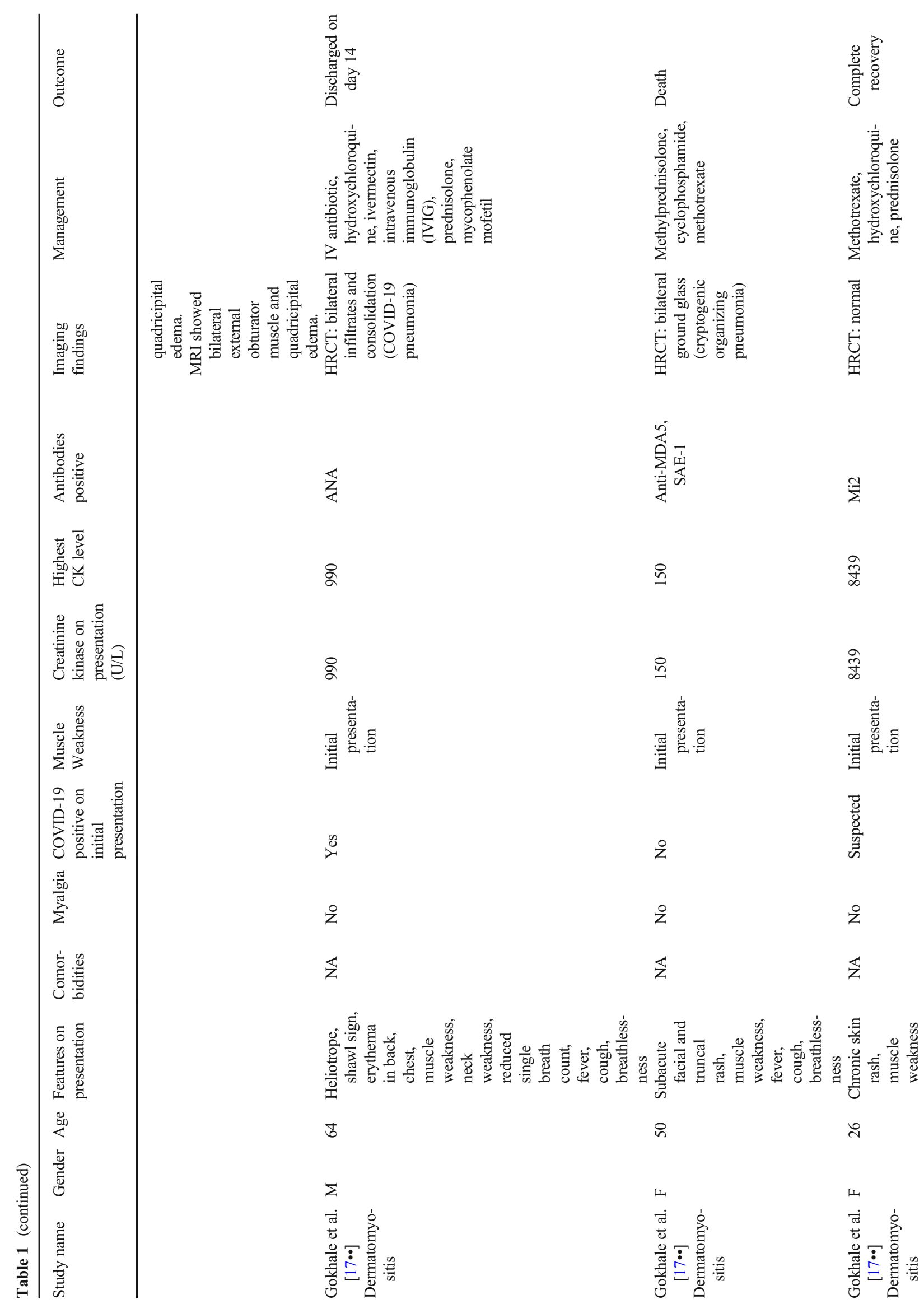




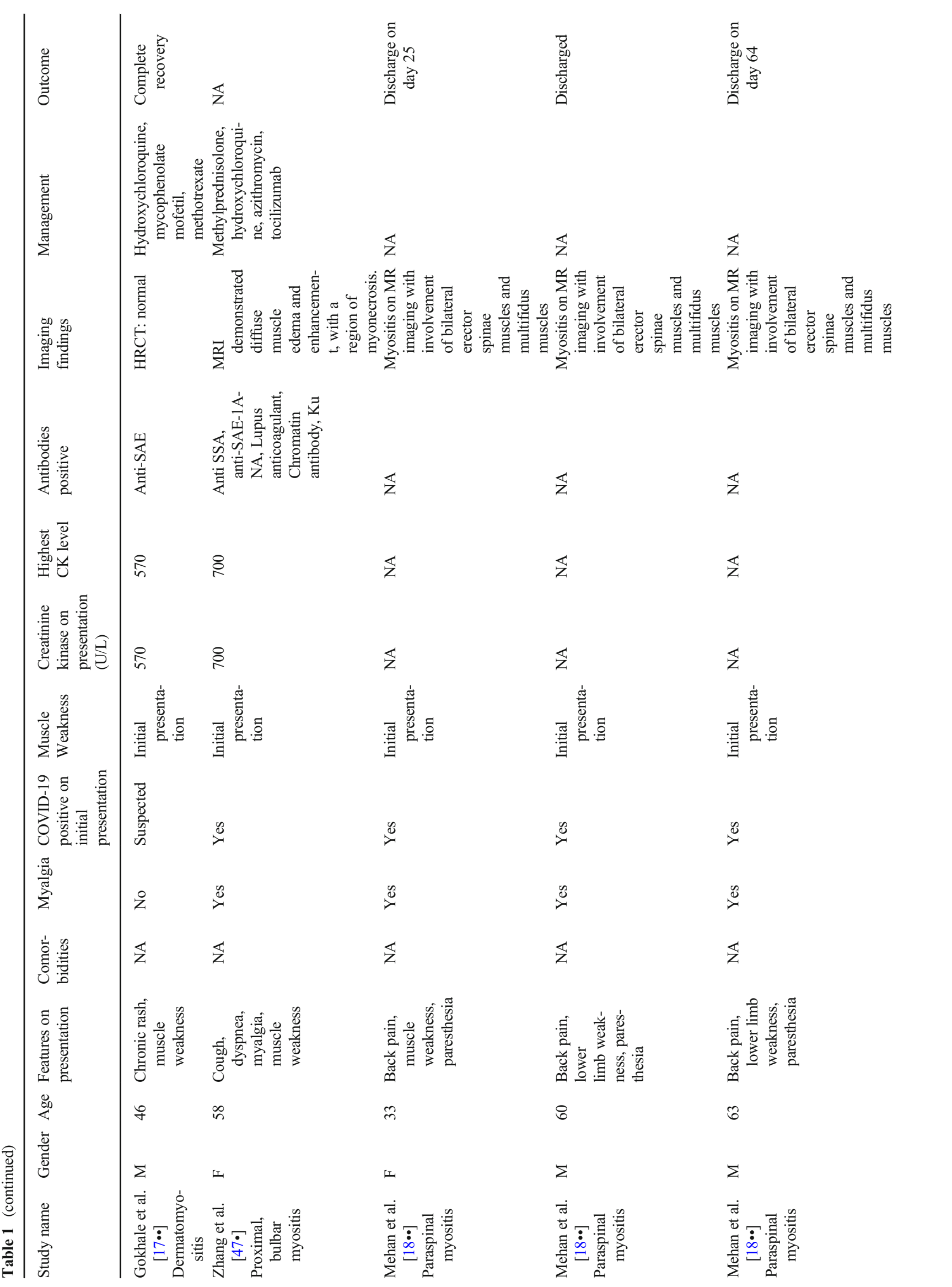




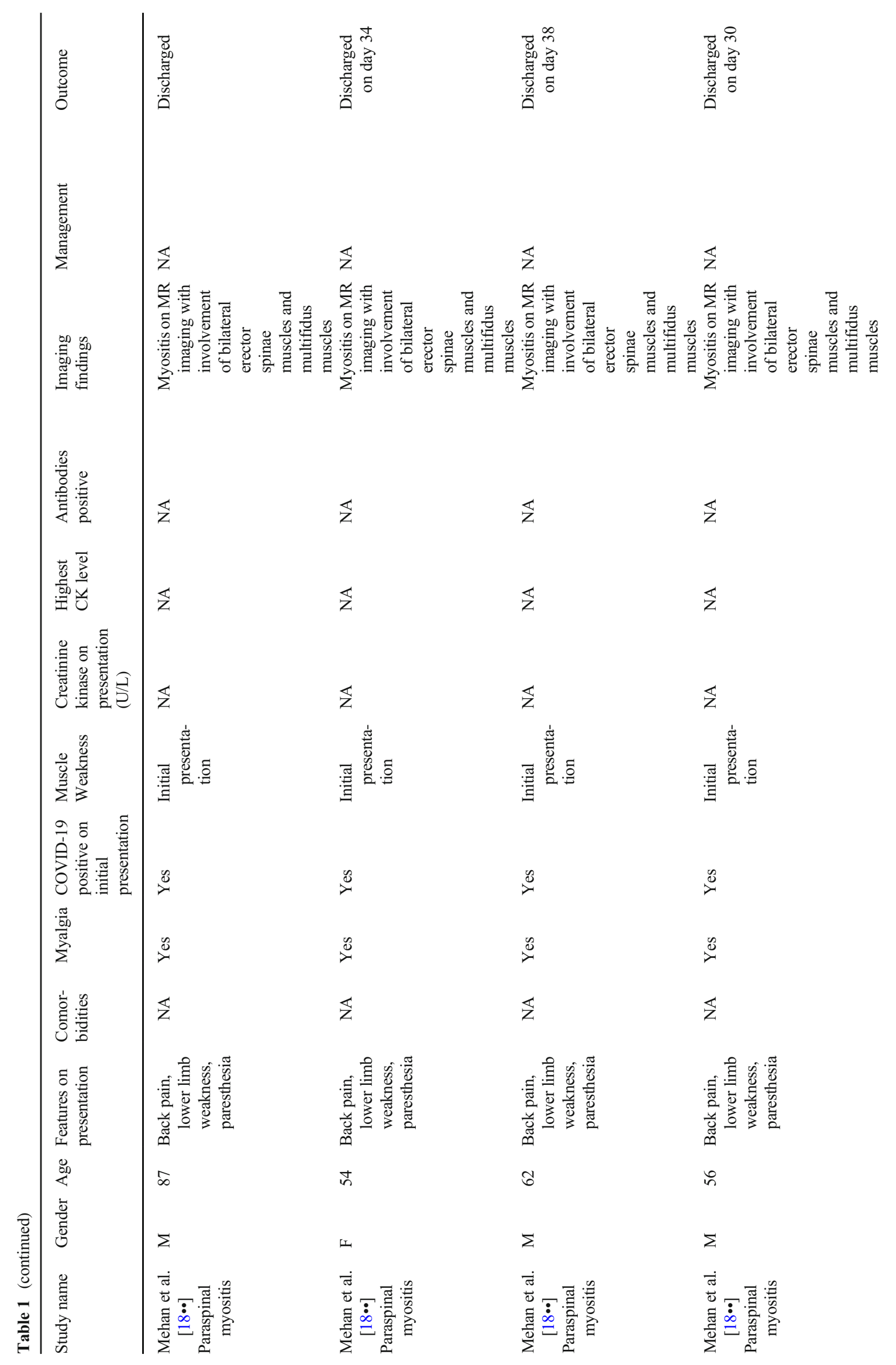


involvement is minimal. Myositis-specific autoantibodies can be an important clue to diagnosis, with recent reports demonstrating anti-Mi2 $(\mathrm{n}=1)$, anti-MDA5 (anti-melanoma differentiation-associated gene 5) $(\mathrm{n}=1)$, anti-SAE1 (antismall ubiquitin-like modifier-1 activating enzyme) $(n=2)$, or anti-nuclear autoantibodies $(\mathrm{n}=1)[17 \cdot \bullet]$. The outcomes may vary, with patients exhibiting milder disease variant regaining muscle strength in a week [17••]. Others had notable long-lasting effects of COVID-19 infection with full strength returning over 3 months. Rarely, the disease can be severe and fatal with severe lung involvement due to COVID-19 infection in the presence of anti-MDA5 antibody which was also reported $[17 \bullet \bullet]$.

\section{Paraspinal Myositis}

Involvement of the erector spinae and multifidus paraspinal muscles has been reported on MRI in those diagnosed with COVID-19 with back pain [18••]. Of the 9 patients of COVID-19 who underwent MRI of the spine, 5 patients had back pain, 2 had bilateral leg pain, 1 had imbalance, and 1 patient had attempted suicide. Notably, muscle edema and enhancement on MRI were always bilateral and occurred exclusively in the lumbar spine over multiple vertebral levels $[18 \cdot \bullet]$. It is noteworthy that paraspinal involvement could be a harbinger for a protracted hospital course spanning over 3 weeks.

Since most patients in the above study had an underlying degenerative spinal disease and occasional neurogenic symptoms necessitating an MRI, neurotropism of SARS-CoV2 could be an interesting hypothesis to explain such observations. SARS-CoV-2 uses the ACE2-mediated endocytotic pathway for internalization and intracellular transport to exploit the retrograde axonal transport machinery, a feature common to most Coronaviruses. It is hypothesized that the virus could potentially disrupt vagal signaling after access to the brainstem through the olfactory nerves. Apart from paraspinal myositis, this model could explain several odd clinical sequelae, including protracted clinical course, neurologic symptoms, and multiple organ affliction even in the absence of lung pathology [19].

\section{Myasthenia as the First Presentation of COVID-19}

Myasthenia may be an early sign of COVID-19 infection, with occurrence around the time of diagnosis or immediately preceding it [20•]. Recent reports suggest a predilection for ocular myasthenia with anti-acetylcholine receptor (AChR) antibody positivity, positive repetitive nerve stimulation test, and fair response to pyridostigmine/intravenous immunoglobulin [20•]. Bulbar myasthenia gravis has also been reported post-COVID-19 infection in the elderly with anti-MuSK (muscle-specific kinase) antibody and a resistant disease 
requiring immunosuppression with azathioprine [21•]. Considering that these patients would likely present to primary care, neurology, or rheumatology, they need to be examined with the differential of COVID-19 in mind.

\section{Asymptomatic Rise in CK}

Elevated CK levels, with other signs of myositis such as rash or weakness, have been reported in as many as $16-33 \%$ of COVID-19 patients [22]. Given that asymptomatic elevated $\mathrm{CK}$ has been associated with increased mortality in some studies, CK should be evaluated in patients with musculoskeletal symptoms in COVID-19 [23, 24].

\section{Other Rarer Manifestations}

Lastly, COVID-19-related axonal neuropathy with residual muscle denervation edema and atrophy is described [25]. On occasion, an isolated group of muscles may be inflamed in patients with COVID-19, resulting in myofascial compartment syndrome, which may even require amputation [26 (Table 1). COVID-19-induced cachexia akin to that seen in cancer patients is also described [27].

\section{Long-term Outcomes in Patients with COVID-19}

In the general population, persistent fatigue and myalgia are emerging as important and functionally debilitating symptoms post-recovery [28]. Data on long-term outcomes for COVID19 infection in rheumatic disease in general and myositis, in particular, is limited and continue to evolve over time.

In cases wherein COVID-19 leads on to acute myositis (Table 1), nearly half have prolonged recovery typically over weeks to months. Cases exhibiting paraspinal myositis have a protracted course with prolonged hospitalization in the majority. In view of the short duration of follow-up reported in most reports, it is currently unclear whether these patients continued to experience weakness, myalgia, relapses, or other musculoskeletal complications after discharge from the hospital. Nevertheless, it seems imperative to closely monitor these individuals for continued musculoskeletal symptoms long after hospital discharge.

\section{COVID-19 in Established IIM}

Environmental factors are important triggers for autoimmune diseases in genetically predisposed individuals and myositis is no exception. Various viral triggers have been identified in patients with myositis, and seasonal variations, as well as viral inclusions in muscle biopsy specimens, further support the hypothesis that viruses may trigger autoinflammation to autoimmunity along a continuum in a conducive genetic environment [29]. While prospective studies on COVID-19 incidence and outcomes in patients with established IIM are awaited, anecdotal evidence supports flares/worsening of disease in the event of COVID-19 infection. Patients with rheumatic disease may be at a higher risk for worse outcomes especially those $>65$ years with diabetes, end-stage renal disease, hypertension, cardiovascular disease, or use of glucocorticoids $>10$ $\mathrm{mg}$ /day prednisolone equivalent [2].

Case reports describe worsened disease activity after COVID-19 infection, in both dermatomyositis and immunerelated necrotizing myositis (IMNM) $[17 \bullet \bullet, 30]$. A 50-yearold male with DM (anti-Mi2 and anti-PM-Scl antibody), presented with flare of myositis and skin rash following COVID19 infection, is one such case reported by Gokhale et al. [17••]. Recently high flare rates in juvenile dermatomyositis (DM) (71\%) in the pandemic period have been described though the relative attribute with COVID-19 infection versus logistic challenges in procuring medicines per se is unclear [3••].

On similar lines, worsening myasthenia crisis can be ascribed to various kinds of infection, and COVID-19 is no exception [31, 32]. In a series of 91 patients with myasthenia gravis, myasthenic worsening required rescue therapy in $40 \%$ who were diagnosed with COVID-19 [33]. Nearly $43 \%$ of them had a complete recovery and were subsequently discharged, but $24 \%$ succumbed to COVID-19. Another study from Sao Paulo confirmed poor outcomes, wherein $87 \%$ of patients with myasthenia gravis were admitted in the intensive care unit due to COVID-19, 73\% needed mechanical ventilation, and $30 \%$ died [34].

\section{COVID-19 - the Human Model for Anti-MDA5 IIM?}

Most types of inflammatory muscle disease have significant clinical muscle weakness. However, forms with hypomyopathic and amyopathic dermatomyositis (clinically amyopathic dermatomyositis (CADM)) are increasingly being recognized. These CADM patients are strongly associated with anti-MDA5 antibody. Anti-MDA5 antibody-positive patients are at high risk for rapidly progressive interstitial lung disease (ILD), which may be more prominent in specific ethnic groups such as the Asian population. In the early pandemic period, immunologists and pulmonologists drew interesting parallels between anti-MDA5-associated rapidly progressive ILD and lung disease of COVID-19, raising several questions regarding a possible pathogenic link.

Clinically, COVID-19 infection may result in remarkably similar chest X-ray findings of bilateral ground-glass opacities, acute respiratory distress syndrome (ARDS), fever, rash, arthralgias, and especially for the purpose of this review, myalgias, heightened CK levels, and pro-inflammatory cytokines. Apart from acute interstitial pneumonia, vasculopathy and thrombosis are notable features of both COVID-19 infection and anti-MDA5 dermatomyositis. The striking similarity 
has forced experts around the world to examine COVID-19 as a potential human model of anti-MDA5 IIM [35••].

Cytokine storm syndrome, well-depicted in COVID-19, is being increasingly recognized in anti-MDA5 DM as well. Fever and hyperferritinemia are also seen both in COVID-19 and in anti-MDA5 DM [35••]. Interestingly, fever is a common manifestation in anti-MDA5 patients compared to those with other connective tissue diseases. Furthermore, bilateral posterior and peripheral ground-glass opacities seen in antiMDA5 are distinctively different from the interstitial lung disease seen in other myositis and similar to the distribution in COVID-19 further suggesting that COVID-19 may be a better model for anti-MDA5 than other connective tissue diseases [36]. Pneumomediastinum is also reported both in COVID-19 and in anti-MDA5 DM but not as prevalent in non-antiMDA5-associated myositis [35••, 36].

Beneath the remarkable similarities between COVID-19 and the anti-MDA5 syndrome may lie a common pathogenic link, wherein the MDA5, an intracellular cytoplasmic viral RNA detector, is activated in both, leading to an intense inflammatory state due to innate immune system activation and interferon production. The MDA5 action is part of the cellular type 1 interferon response axis in response to viral pathogen and other triggers $[17 \bullet \bullet]$. Worth noting is the fact that IFN- $\alpha$ is a mechanism of viral defense [37], and hence, it is an important consideration that viral infection, possibly COVID-19, may be implicated in anti-MDA5 dermatomyositis as well. Several reports implicate cytomegalovirus activation and other environmental infectious drivers in anti-MDA5-positive IIM [36, 38].

The similarities between the conditions hold implications for targeted treatment development, with agent like human immunoglobulins, high-dose corticosteroids, JAK (Janus kinase) inhibitors, and $\mathrm{T}$ cell modulators currently in trials and IL-6 inhibitors (e.g., tocilizumab), IL-1 inhibitors (e.g., anakinra), anti-GM-CSF (gimsilumab), and anti-IFN $\gamma$ agents (e.g., emapalumab) currently being looked as potential for treatment of COVID-19 [36]. Drawing parallels between the two diseases can help guide better therapeutic strategies in combatting the post-inflammatory states of both these conditions. Furthermore, regression of anti-MDA5 IIM as well as COVID-19 with glucocorticoids as well as tofacitinib in recent times further substantiates the striking overlap between the two conditions [39]. At a time where the debate regarding the risk vs. benefit of the use of immunosuppression in patients with diagnosed COVID-19 is still brewing, Tacrolimus, which has been successfully used in anti-MDA5-positive disease, shows promise in controlling COVID-19 replication and needs to be examined further [40].

The stark similarity in presentation and response to immunosuppressive therapy highlights the need to further examine the MDA5 functionality in COVID-19-infected patients with IIM. Notably, emerging reports of underlying unidentified genetic defects in the interferon signaling axis, including the IFIH/MDA5 gene and various autoantibodies in COVID-19, further lend support to this hypothesis [41]. A recent case series described two new cases of anti-MDA5-associated IIM possibly triggered after COVID-19 infection, further substantiating an intersection between the two diseases (Table 1).

\section{Proposed Pathogenesis of COVID-19-Triggered Muscle Inflammation}

\section{Direct Virus Entry into the Muscle via ACE-2 Receptors}

Akin to most other sites predisposed to SARS-CoV-2, the muscle tissue expresses ACE-2 receptors as well [6]. Extrapulmonary manifestations of COVID-19 are thought to occur through ACE-2 receptor-mediated facilitation of virus entry into small vessel endothelium, organs like the bowel, synovial tissue, and both smooth and skeletal muscles [42]. In effect, this may be the reason behind multisystem involvement with COVID-19. It has been speculated that COVID-19induced myositis may also transpire via direct muscle entry of the virus through these receptors on the muscle cells. ${ }^{38}$ Specifically, the viral spike protein attachment to the ACE-2 receptor may enable SARS-CoV-2 viral envelope coupling with the host cell membrane with the genetic material transfer into the cell. Notably, it is suggested that SARS-CoV-2 may be the first virus capable of infecting muscle fibers directly [43].

\section{Virus-Triggered Innate Immune Activation/Autoinflammation}

Most other virus-associated muscle inflammation is attributed to T-cell clonal expansion and the production of proinflammatory cytokines resulting in muscle damage [7]. It seems plausible that these mechanisms may also be operative in COVID-19 as well. Specifically, SARS-CoV-2 may bind and activate TLR4 to increase ACE2 expression, facilitating entry and causing hyperinflammation as recorded in other inflamed tissues [44].

\section{Virus-Triggered Adaptive Immune Activation}

Recently, the identification of 3 different $\mathrm{T}$ cell receptor epitopes "highly specific" for SARS-CoV-2 (O-ribose methyltransferase, RNA-dependent RNA polymerase, and $3^{\prime}-$ to- $^{\prime}{ }^{\prime}$ exonuclease proteins) in dermatomyositis patients suggested that the virus may be a trigger for CD8 T-cell overactivation and lead on to COVID-19-induced myositis [45••]. 


\section{Other Proposed Mechanisms}

Several other mechanisms have been postulated and proposed in the immune-mediated muscle injury observed in COVID19 infection. An alternate or co-existing mechanism for myositis in COVID-19 pathogenesis may include the induction of a hyper-inflammatory state with a rise in TNF-alpha, interferon-alpha, interleukin-1, and interleukin-6 among other cytokines [46]. The stimulation of inflammation following the positing of antigen-antibody complexes is one of the mechanisms proposed [17••, 47•]. Another mechanism includes antibody attack of the myocytes as a result of cross-reactivity where antibodies initially produced against the virus attack muscle cells due to molecular mimicry leading to B-cell and T-cell activation and the creation of a hyper-inflammatory state with subsequent myocyte injury. Another mechanism is the direct intake of viral antigen by the myocytes, leading to structural deformity of the myocyte [48].

\section{Impact of COVID-19 Pandemic on the Care of Patients with Established IIM}

Social distancing restrictions and public-health laws limiting the extent of in-person visits have completely altered the dynamics of health care provisions during the COVID-19 pandemic. The pandemic period also saw a switch to teleconsultations, with patients with myositis requiring disproportionately greater admissions as compared with other rheumatic diseases $[4 \bullet \bullet, 49]$. Hence, now, telemedicine is being increasingly utilized to provide care to patients in this new era. With over half of IIM patients availing telemedicine consultations, remote monitoring has become the modality of choice for continuity of care in IIM patients (preferred by up to 70\%) with in-person visits to the health care provider falling out of favor (preferred by only about $25 \%$ ) in these patients [4・•] [50•]. This is understandably due to fears of acquiring the viral infection and going into public spaces $[3 \bullet \bullet, 51 \bullet \cdot$. Global studies, such as one conducted in Poland also show marked patient approval for virtual consultations over in-person visits [52].

\section{Challenges}

The COVID-19 pandemic has presented many obstacles in the provision of care to many patients around the globe. This is especially true for autoimmune diseases like IIM where patients ideally require continual ongoing check-ups, examinations, and frequent medication reviews. With the disruption of the global health care framework, modified allocation and distribution of health care resources have negatively impacted IIM patients. In an e-Survey conducted among 608 IIM patients, $32.1 \%$ of respondents reported having one or more issues relating to their condition during the COVID-19 pandemic $[51 \bullet \bullet]$. Half of these patients had medicationrelated issues, requiring an increase in medication dose with $13 \%$ requiring glucocorticoid dose elevation due to ineffective disease control. Also, it is important to note that over half were already on glucocorticoid treatment which may increase COVID-19 susceptibility, complications, and hospitalization rates in rheumatologic patients [53]. About $18 \%$ of patients with IIM were hospitalized for disease-related complications, while a fifth experienced delay in receiving infusion treatment necessary for their condition [51••]. Furthermore, $30.7 \%$ experienced symptoms that were of unclear origin further presenting challenges to management during this time. Another obstacle reported by patients in their management was the difficulty in procuring medication $(26.3 \%)$ and while a majority were able to get medications delivered by family members or alternative means, $10.6 \%$ many of these patients were still unable to obtain medication forcing a temporary halt in their treatment regimen $[3 \bullet \cdot, 51 \bullet \bullet]$.

While telemedicine may be the saving grace in the eyes of both doctors and patients alike, it must be noted that even this brings with it its own challenges with inadequate lighting, poor video or audio quality, lack of thorough examination, etc., limiting ideal care to patients during this time, and methods to resolve these issues need to be examined. Moreover, it may specifically disenfranchise poor or elderly, who may not have the means or not technologically savvy, to get appropriate care through telemedicine.

In developing countries, the provision of telemedicine care to financially challenged groups unable to afford reliable internet connections or quality smart phones continues to remain a challenge in maintaining adequate disease control. This is compounded by the markedly lower educational as well as technological literacy rates in these countries [3••]. Furthermore, physiotherapy forms an important component of IIM management, yet one in three patients was not able to receive timely physical therapy sessions during pandemic [51・•]. This is especially of concern because deconditioning in patients with sarcopenia/muscle disease has been reported as a significant but preventable complication amenable with timely detection and physical and/or occupational therapy [54].

\section{Solutions}

While not much can replace the effectiveness of in-person physical examinations in assessing patients, several authors have proposed solutions and ideas for combating the challenges presenting during these difficult times. The need to shift to virtual consultations has been highlighted by many authors $[4 \bullet \bullet, 51 \bullet, 55]$. Transition to the use of mobile applications to determine patient self-reported outcomes and track symptoms may improve patient management in cases with limited in-person interaction between patient and doctor $[3 \bullet \bullet$, 
51••]. A need has been highlighted to develop accurate and approved outcome measures with high sensitivity for disease progression and control in patients with IIM. Self-physical assessment of patient at home with 2-min walking-distance test (meters walked), timed up and go (time taken to stand, walk for $10 \mathrm{~m}$, turn back and return to chair), 30-s sit to stand (number of repetitions of sit to stand in $30 \mathrm{~s}$ ), and an arm-raise test (time taken to raise arms above head 10 times) have been some of the serial-outcome measures that can be employed for remote monitoring [3*0]. In addition, daily monitoring of symptoms and validated patient-reported outcomes can enhance care of patient and may lead to early detection and intervention of flare or worsening of the disease [56, 57]. Such routine monitoring tests are especially important because approximately a quarter of COVID-19 patients show a decrease in 6-min walking distance even after 6 months of hospital discharge suggesting long-term persistence of COVID19-related musculoskeletal complications [54]. It has been proposed that applications reporting self-outcome measures should be internally linked to national health care databases which will help expedite care at a time when clinicians are finding it difficult to manage the workload in order to care for the maximum number of patients at the best standard of care reasonably possible, especially in chronic autoimmune diseases $[51 \bullet, 55]$. Studies reporting no difference in patient satisfaction between those managed in clinics and those managed via remote consultations are positive signs suggesting that telemedicine that has entrenched its place in patient management will play a vital role not only during this pandemic but possibly in the post-COVID-19 era as well [3••].

In regard to mobile programs, applications like WhatsApp secured messaging and video chat are finding a use for answering quick patient queries. The type of application used varies from country to country with WeChat finding use in China $[3 \bullet \bullet, 58]$. Furthermore, to combat long delays in infusion delivery to patients, home-administered subcutaneous injections have been proposed, and an attempt to determine its

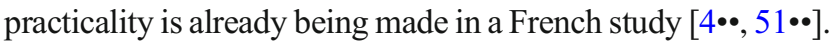
Provision of video-assisted physiotherapy, dietary advice, mindfulness, and yoga sessions on a continuous basis to patients with long-standing IIM may be greatly beneficial in maintaining disease control [3••]. At-home medication delivery via pharmacies or intermediary courier service can tackle the issue of drug procurement experienced by many IIM patients $[51 \bullet \cdot]$.

Mental health and anxiety management is yet another sector that needs to be analyzed and addressed with an increasingly important role of cognitive behavioral therapy in combating this increasingly prevalent issue [ $51 \bullet \bullet]$. It is also important to note that this is an issue that does not simply vanish with the regression of the disease, and patients who have had myositis or COVID-19 may still have anxiety and depression long after the disease is under control [54]. Considering how mental health issues may highlight a separate need for triaging patients into those who may benefit from further intervention, expanding telecommunication to the to the primary-care sector may be crucial in ensuring that these patients receive adequate counseling and mental health support during these times.

\section{Need for Tele-triage Which May Be Greater in IIM During the Pandemic}

With the advent of a global pandemic that poses a health risk to patients and healthcare workers alike, teleconsultations have become a staple of patient care and management. The realization that not all patients will present with the same severity of disease or require admission has helped accelerate patient care by stratifying patients requiring hospital admission from those that can be managed conservatively after a video teleconsultation, a method called tele-triaging which has recently been gaining attention $[3 \cdot \bullet, 19]$. An increasing need for tele-triaging patients was felt during these times and holds the potential for better management of patient relapses through effective risk stratification and telecommunication [3••]. This will help ensure that patients who can be managed with medication receive timely medication delivered at home, and only those who require urgent admission get admitted $[3 \cdot \bullet]$. The ultimate goal of such triaging would be to reduce the healthcare burden and ensuring that adequate bed spaces remain vacant for those people who genuinely require them, and at this time, especially those infected by COVID-19 and suffering from its complications, so that they can be treated promptly.

\section{Unresolved Questions Raised in COVID-19-Associated Myositis}

\section{Is Diagnostic Testing for Myositis Warranted in Patients Presenting with COVID-19 and Myalgias?}

A gray area that remains still is the need for and importance of modalities like muscle biopsies, electromyograms, and MRIs in the diagnosis of patients. Currently available studies on COVID-19-associated myositis show remarkably variability in CK on the first presentation. In a case series by Gokhale et al., a patient diagnosed with dermatomyositis had normal CK levels but died because of COVID-19. However, another patient with a greatly elevated CK of $42,670 \mathrm{IU} / \mathrm{L}$ had a favorable recovery within 9 days. In light of such studies, it then seems reasonable to assume, at least on a preliminary analysis of cases, that $\mathrm{CK}$ elevation does not correlate directly with disease severity in all patients with IIM and that myositisspecific clinical signs and symptoms by thorough history and examination as well as markers such as myositis- 
specific autoantibodies such as anti-MDA5, inflammatory markers (C-reactive protein (CRP) and erythrocyte sedimentation rate (ESR)), etc. may need to be taken into consideration together with CK levels to determine disease severity in patients presenting with IIM.

Views on the use of muscle biopsies remain heterogeneous. Several authors cite the potential risks of biopsies such as prolonged bleeding and increased recovery times as deterrence for use of this diagnostic modality in the majority of patients [12•, 59-61]. Similarly, others do not advocate the need for muscle biopsies in COVID-19 patients with myalgias, stating that a clinical diagnosis is sufficient in the presence of a diagnosed viral infection $[47 \cdot, 62,63]$. In contrast, Almadani et al. reports a case of COVID-19-associated myositis complicated by compartment syndrome, and Bolig et al. a case of immune-mediated necrotizing myopathy, where both felt that a muscle biopsy was warranted $[26 \bullet, 30]$. Hence, muscle biopsies may be warranted in certain specific situations where myositis associated with COVID-19 is complicated by a more sinister problem.

\section{Immunosuppressive Treatment in Myositis; a Double- edged Sword?}

Immunosuppressive medications are the treatment of choice for most inflammatory myopathies; however, they lead to detriment of the body's immunological capabilities which are required to fend off infectious agents. Hence, the topic of whether to continue or withhold immunosuppressive treatment in COVID-19 patients with myositis currently remains a hot-topic up for debate.

An interesting case of an initially well-suppressed immunemediated necrotizing myopathy has been reported where the IMNM recurred after mycophenolate mofetil and intravenous immunoglobulins were withheld for 6 and 8 weeks, respectively, following a diagnosis of COVID-19 [30]. This led to the suggestion that holding immunosuppressants may lead to a recurrence of myositis flares and thus not necessarily need to be stopped in people with inflammatory myopathies with COVID-19 [30]. Although immunosuppressive treatment may be warranted in patients with this subset of myositis, a fine balancing act has to be taken into consideration as immunosuppression can lead to worsening of COVID-19 complications [64]. Mycophenolate mofetil results in T-cell and Bcell suppression; however, studies have reported some antiviral effects both in vitro and in vivo [65]. Another unexplored area is the severity of COVID-19 in patients with myositis -who have activated interferon axis which may potentially help them clear the virus better. However, this is subject to various confounders, and until more research is made available, a case-by-case approach and individualized treatment plan may be necessary for managing these patients.
A high level of concern however must always be maintained while a patient is on immunosuppressants as death has been observed in a patient on prednisolone and cyclophosphamide for dermatomyositis (Table 1, 17). What the authors suggest is not the complete abolishment of immunosuppressives in COVID-19 patients with myositis, but the increasing need to monitor medication, especially switching from highly immunosuppressive agents to less immunosuppressive agents. Furthermore, in cases where there were two potential treatments available, such as IVIG and rituximab for bulbar palsy, IVIG was preferred as it provided concomitant protection against COVID-19 pneumonia as well, while for MDA-5associated dermatomyositis with interstitial lung disease, cyclophosphamide was the preferred choice over rituximab due to its shorter duration of actions [17••]. These small considerations in medication management during the times of COVID19 are critically important, especially where immunosuppression can be a matter of life and death for patients with IIMs.

Furthermore, emerging data from cohort studies seem to suggest a beneficial role of agents like tocilizumab and hydroxychloroquine, which, by their immune modulation, dampen the inflammatory response and consequently reduce morbidity by preventing cytokine storms [19]. Taken together in the light of the findings claiming higher COVID-19 morbidity in immunocompromised patients, such a statement seems contradictory, but harbors support from other authors as well who bring the benefits of agents like tocilizumab to the fore.

\section{Frequency, Severity, and Outcomes of COVID-19 in IIM}

While rheumatic diseases incur a higher risk of adverse outcomes in COVID-19, the exact burden of morbidity, mortality, attributable risk for adverse outcomes, and risk factors for mortality is to be ascertained. It seems plausible that besides conventional risk factors for COVID-19, immunosuppression, underlying disease damage, and organ involvement such as ILD may contribute to COVID-19-related morbidity in IIM.

\section{COVID-19 as an Environmental Risk Factor for the Onset of IIM}

Another unexplored area is the possibility of an increase in the incidence of IIM post-pandemic, with the emergence of virustriggered myositis in the genetically predisposed. Many of these individuals may potentially harbor minor immune pathway defects, placing them on the helm of environmental trigger leading to increase frequency and or severity of autoimmunity.

To conclude, COVID-19 can induce myositis, potentially by direct infection of the muscle, and as an inciting environmental event triggering autoimmunity. The clinical profile can 
be highly varied, warranting a high index of suspicion for myositis in any COVID-19 patient. A greater recognition of the stark similarity between anti-MDA5-positive myositis with COVID-19 has thrown researchers into the alley of exploration - finding common etiopathogenic basis as well as therapeutic strategies. With immunosuppression seen both a friend and a foe, we certainly need more data from large registry-based prospective studies to help physicians better manage patients with IIM in the setting of COVID-19 infection. While the COVID-19 pandemic may be behind us soon, the massive effects of the pandemic including disease damage due to fragmented care, fear, and anxiety, as well as innovative healthcare delivery such as teleconsultation, may be here to stay. Embracing these new means of communication and adapting to new models of tele-triage and remote patient disease monitoring through patient-reported and patient-oriented outcome measures may be the way forward.

Abbreviations ACE2, Angiotensin-converting enzyme 2 receptor; Anti-MDA 5, Anti-melanoma differentiation-associated gene 5; AntiSAE1, Anti-small ubiquitin-like modifier-1 (SUMO 1)-activating enzyme; Anti-MUSK, Anti-muscle-specific kinase; ARDS, Acute respiratory distress syndrome; CK, Creatine kinase; CT, Computerized tomography; DM, Dermatomyositis; IFN 1, Type 1 interferons; IIM, Idiopathic inflammatory myopathies; IMNM, Immune-mediated necrotizing myopathy; MG, Myasthenia gravis; MRI, Magnetic resonance imaging

Author Contribution All authors collected and analyzed the data. All authors were involved in writing and reviewing the manuscript for critical intellectual inputs.

\section{Declarations}

Conflict of Interest The authors declare no competing interests.

Human and Animal Rights Informed Consent This article does not contain any studies with human or animal subjects performed by any of the authors.

\section{References}

Papers of particular interest, published recently, have been highlighted as:

- Of importance

•- Of major importance

1. Galeotti C, Bayry J. Autoimmune and inflammatory diseases following COVID-19. Nat Rev Rheumatol. 2020 Aug;16(8):413-4.

2. Hyrich KL, Machado PM. Rheumatic disease and COVID-19: epidemiology and outcomes. Nat Rev Rheumatol. 2021 Feb;17(2): $71-2$.

3.• Naveen R, Sundaram TG, Agarwal V, Gupta L. Teleconsultation experience with the idiopathic inflammatory myopathies: a prospective observational cohort study during the COVID-19 pandemic. Rheumatol Int. 2021;41(1):67-76. In a cohort of IIM, onethirds resorted to voice-based teleconsultation services on an emergency basis. Over a quarter reported relapse, these being eight times more common in JDM than adult IIM.

4.• Gupta L, Misra DP, Agarwal V, Balan S, Agarwal V. Response to: 'Telerheumatology in COVID-19 era: a study from a psoriatic arthritis cohort' by Costa et al. Ann Rheum Dis. 2020:annrheumdis2020-217953. Patients with IIM were underrepresented among teleconsultations but overrepresented among hospital admissions in the pandemic period, suggesting the need for a teletriage protocol based on the type of rheumatic disease.

5. Zuo Y, Estes SK, Ali RA, Gandhi AA, Yalavarthi S, Shi H, et al. Prothrombotic autoantibodies in serum from patients hospitalized with COVID-19. Sci Trans1 Med. 2020;12(570):eabd3876.

6. Paliwal VK, Garg RK, Gupta A, Tejan N. Neuromuscular presentations in patients with COVID-19. Neurol Sci. 2020;41(11):303956.

7. Huang C, Wang Y, Li X, Ren L, Zhao J, Hu Y, et al. Clinical features of patients infected with 2019 novel coronavirus in Wuhan. China The Lancet. 2020;395(10223):497-506.

8. Madjid M, Safavi-Naeini P, Solomon SD, Vardeny O. Potential effects of coronaviruses on the cardiovascular system: a review. JAMA Cardiol. 2020;5(7):831-40.

9. Talotta R, Robertson E. Autoimmunity as the comet tail of COVID19 pandemic. World J Clin Cases. 2020;8(17):3621-44.

10. Jin M, Tong Q. Rhabdomyolysis as potential late complication associated with COVID-19. Emerg Infect Dis. 2020;26(7):161820.

11. Suwanwongse K, Shabarek N. Rhabdomyolysis as a presentation of 2019 novel coronavirus disease. Cureus [Internet]. 2020 Apr 6 [cited $2021 \mathrm{Feb} 10]$. Available from: https://www.cureus.com/ articles/30228-rhabdomyolysis-as-a-presentation-of-2019-novelcoronavirus-disease

12. Husain R, Corcuera-Solano I, Dayan E, Jacobi AH, Huang M. Rhabdomyolysis as a manifestation of a severe case of COVID19: a case report. Radiol Case Rep. 2020;15(9):1633-7.

13. Singh B, Kaur P, Mechineni A, Maroules M. Rhabdomyolysis in COVID-19: report of four cases. Cureus [Internet]. 202027 [cited $2021 \mathrm{Feb} 10]$; Available from: https://www.cureus.com/articles/ 41837-rhabdomyolysis-in-covid-19-report-of-four-cases

14. Taxbro K, Kahlow H, Wulcan H, Fornarve A. Rhabdomyolysis and acute kidney injury in severe COVID-19 infection. BMJ Case Rep. 2020 Sep;13(9):e237616.

15. Beydon M, Chevalier K, Al Tabaa O, Hamroun S, Delettre A-S, Thomas M, et al. Myositis as a manifestation of SARS-CoV-2. Ann Rheum Dis. 2020; annrheumdis-2020-217573.

16. Zhang H, Charmchi Z, Seidman RJ, Anziska Y, Velayudhan V, Perk J. COVID-19-associated myositis with severe proximal and bulbar weakness. Muscle Nerve [Internet]. 2020 Sep [cited 2021 Feb 10];62(3). Available from: https://onlinelibrary.wiley.com/doi/ abs/10.1002/mus.27003

17••. Gokhale Y, Patankar A, Holla U, Shilke M, Kalekar L, Karnik ND, et al. Dermatomyositis during COVID-19 pandemic (a case series): is there a cause effect relationship? J Assoc Physicians India. 2020;68(11). This series describes four new cases of typical dermatomyositis where the onset of the illness was preceded by COVID-19 infection, raising the possibility of a direct pathogenic link.

18.• Mehan WA, Yoon BC, Lang M, Li MD, Rincon S, Buch K. Paraspinal myositis in patients with COVID-19 infection. Am J Neuroradiol. 2020;41(10):1949-52. The importance of this article is for its reporting of para-spinal myositis cases which helps describe this rare complication not commonly reported in the literature following COVID-19 infection as well as reemphasizing the extent and pervasiveness of the myositis associated with this disease.

19. Ferri C, Giuggioli D, Raimondo V, L'Andolina M, Tavoni A, Cecchetti R, et al. COVID-19 and rheumatic autoimmune systemic 
diseases: report of a large Italian patients series. Clin Rheumatol. 2020 Nov;39(11):3195-204.

20. Sriwastava S, Tandon M, Kataria S, Daimee M, Sultan S. New onset of ocular myasthenia gravis in a patient with COVID-19: a novel case report and literature review. J Neurol [Internet]. 2020 Oct 12 [cited $2021 \mathrm{Feb} 10$ ]; Available from: http://link.springer. com/10.1007/s00415-020-10263-1

21. Assini A, Gandoglia I, Damato V, Rikani K, Evoli A, Del Sette M. Myasthenia gravis associated with anti-MuSK antibodies developed after SARS-CoV-2 infection. Eur J Neurol. 2021; ene.14721.

22. Guan W, Ni Z, Hu Y, Liang W, Ou C, He J, et al. Clinical characteristics of coronavirus disease 2019 in China. N Engl J Med. 2020;382(18):1708-20.

23. Rivas-García S, Bernal J, Bachiller-Corral J. Rhabdomyolysis as the main manifestation of coronavirus disease 2019 . Rheumatology. 2020;59(8):2174-6.

24. Zhou F, Yu T, Du R, Fan G, Liu Y, Liu Z, et al. Clinical course and risk factors for mortality of adult inpatients with COVID-19 in Wuhan, China: a retrospective cohort study. Lancet. 2020;395(10229):1054-62.

25. Bahouth S, Chuang K, Olson L, Rosenthal D. COVID-19 related muscle denervation atrophy. Skeletal Radiol [Internet]. 2021 Jan 31 [cited 2021 Feb 10]; Available from: http://link.springer.com/10. 1007/s00256-021-03721-y

26. Almadani M, Shiferson A, Swearingen B, Shih M, Jacob T, Rhee R. Compartment syndrome secondary to viral myositis as initial presentation in COVID-19 patient. J Vasc Surg Cases Innov Tech. 2020 Dec;6(4):524-7.

27. Kumar NB. Does COVID-19-related cachexia mimic cancerrelated cachexia? Examining mechanisms, clinical biomarkers, and potential targets for clinical management. J Cachexia Sarcopenia Muscle. 2021; jcsm.12681.

28. Carfi A, Bernabei R, Landi F, for the Gemelli Against COVID-19 Post-Acute Care Study Group. Persistent symptoms in patients after acute COVID-19. JAMA. 2020;324(6):603.

29. Vegosen LJ, Weinberg CR, O'Hanlon TP, Targoff IN, Miller FW, Rider LG. Seasonal birth patterns in myositis subgroups suggest an etiologic role of early environmental exposures. Arthritis Rheum. 2007;56(8):2719-28.

30. Bolig TC, Abdulaziz N, Schiopu E. Complete recovery from COVID-19 bilateral pneumonia in an immunosuppressed man with immune-mediated necrotizing myopathy. Schiavon F, editor. Case Rep Rheumatol. 2020 Oct 21;2020:1-5.

31. Fares E, Tayyar R, Pathak K, Damiano C, Kuntz C. Myasthenia gravis crisis triggered by COVID-19. Chest. 2020 Oct;158(4): A734.

32. Aksoy E, Oztutgan T. COVID-19 presentation in association with myasthenia gravis: a case report and review of the literature. Case Rep Infect Dis. 2020;2020:1-4.

33. Muppidi S, Guptill JT, Jacob S, Li Y, Farrugia ME, Guidon AC, et al. COVID-19-associated risks and effects in myasthenia gravis (CARE-MG). Lancet Neurol. 2020 Dec;19(12):970-1.

34. Camelo-Filho AE, Silva AMS, Estephan EP, Zambon AA, Mendonça RH, Souza PVS, et al. Myasthenia gravis and COVID-19: clinical characteristics and outcomes. Front Neurol. 2020;11:1053.

35.• Giannini M, Ohana M, Nespola B, Zanframundo G, Geny B, Meyer A. Similarities between COVID-19 and anti-MDA5 syndrome: what can we learn for better care? Eur Respir J. 2020;56(3): 2001618. This study draws striking parallels between antiMDA5 syndrome (an autoimmune disease) and COVID-19 which both present with remarkably similar presentations suggesting that future studies should further examine whether there are similar pathological autoimmune mechanisms or pathways involved in both diseases. The potential role of corticosteroids in treating COVID-19 is discussed as corticosteroids have shown benefit in anti-MDA5 syndrome.

36. De Lorenzis E, Natalello G, Gigante L, Verardi L, Bosello SL, Gremese E. What can we learn from rapidly progressive interstitial lung disease related to anti-MDA5 dermatomyositis in the management of COVID-19? Autoimmun Rev. 2020 Nov;19(11):102666.

37. Morris AG. Interferons. Immunol Suppl. 1988;1:43-5.

38. Sato S, Hoshino K, Satoh T, Fujita T, Kawakami Y, Fujita T, et al. RNA helicase encoded by melanoma differentiation-associated gene 5 is a major autoantigen in patients with clinically amyopathic dermatomyositis: Association with rapidly progressive interstitial lung disease. Arthritis Rheum. 2009 Jul;60(7):2193-200.

39. Alunno A, Najm A, Machado PM, Bertheussen H, Burmester GR, Carubbi $\mathrm{F}$, et al. EULAR points to consider on pathophysiology and use of immunomodulatory therapies in COVID-19. Ann Rheum Dis. 2021; annrheumdis-2020-219724.

40. Schoot TS, Kerckhoffs APM, Hilbrands LB, van Marum RJ. Immunosuppressive drugs and COVID-19: a review. Front Pharmacol. 2020;11:1333.

41. Elhabyan A, Elyaacoub S, Sanad E, Abukhadra A, Elhabyan A, Dinu V. The role of host genetics in susceptibility to severe viral infections in humans and insights into host genetics of severe COVID-19: A systematic review. Virus Res. 2020;289:198163.

42. Ciaffi J, Meliconi R, Ruscitti P, Berardicurti O, Giacomelli R, Ursini F. Rheumatic manifestations of COVID-19: a systematic review and meta-analysis. BMC Rheumatol. 2020;4(1):65.

43. Dalakas MC. Guillain-Barré syndrome: The first documented COVID-19-triggered autoimmune neurologic disease: more to come with myositis in the offing. Neurol - Neuroimmunol Neuroinflammation. 2020 Sep;7(5):e781.

44. Aboudounya MM, Heads RJ. COVID-19 and Toll-like receptor 4 (TLR4): SARS-CoV-2 may bind and activate TLR4 to increase ACE2 expression, facilitating entry and causing hyperinflammation. In: Dozio E, editor. Mediators Inflamm. 2021 Jan 14;2021:1-18.

45.• Megremis S, Walker TDJ, He X, Ollier WER, Chinoy H, Hampson $\mathrm{L}$, et al. Antibodies against immunogenic epitopes with high sequence identity to SARS-CoV-2 in patients with autoimmune dermatomyositis. Ann Rheum Dis. 2020;79(10):1383-6. This study found immunogenic epitopes in patients with dermatomyositis that resemble epitopes from SARS-CoV-2. These epitopes are believed to activate CD8 $T$ cells and should guide further studies looking at inflammatory mechanisms in COVID-19-induced myositis.

46. Thepmankorn P, Bach J, Lasfar A, Zhao X, Souayah S, Chong ZZ, et al. Cytokine storm induced by SARS-CoV-2 infection: the spectrum of its neurological manifestations. Cytokine. 2021 Feb;138: 155404.

47. Zhang Q, Shan KS, Minalyan A, O'Sullivan C, Nace T. A rare presentation of Coronavirus Disease 2019 (COVID-19) induced viral myositis with subsequent rhabdomyolysis. Cureus [Internet]. 2020 May 12 [cited 2021 Feb 10]; Available from: https://www. cureus.com/articles/31422-a-rare-presentation-of-coronavirusdisease-2019-covid-19-induced-viral-myositis-with-subsequentrhabdomyolysis

48. Naylor CD, Jevnikar AM, Witt NJ. Sporadic viral myositis in two adults. CMAJ Can Med Assoc J J Assoc Medicale Can. 1987 Nov 1;137(9):819-21.

49. Gupta L, Gupta P. Telecommunication in the Covid19 era: as an assessment tool for patient with dermatomyositis. Indian J Rheum 2021. [in press]. 2021.

50. Gupta L, Misra DP, Agarwal V, et al. Management of rheumatic diseases in the time of covid-19 pandemic: perspectives of rheumatology practitioners from India. Ann Rheum Dis 2020. doi:10.1136/ annrheumdis-2020-217509. [Epub ahead of print: 16 Apr 2020]. 
51.• Gupta L, Lilleker JB, Agarwal V, Chinoy H, Aggarwal R. COVID19 and myositis - unique challenges for patients. Rheumatology. 2021;60(2):907-10. COVID-19 has incurred a detrimental effect on patients with myositis, with widespread difficulties in procuring medicines, delayed biologic infusions, and disrupted physiotherapy sessions. Such delays and omissions in clinical care may translate to poorer outcomes in the future.

52. Opinc A, Łukasik Z, Makowska J. The attitude of Polish rheumatology patients towards telemedicine in the age of the COVID-19 pandemic. Reumatologia/Rheumatology. 2020;58(3):134-41.

53. Kaiser UB, Mirmira RG, Stewart PM. Our response to COVID-19 as endocrinologists and diabetologists. J Clin Endocrinol Metab. 2020;105(5):1299-301.

54. Huang C, Huang L, Wang Y, Li X, Ren L, Gu X, et al. 6-month consequences of COVID-19 in patients discharged from hospital: a cohort study. Lancet. 2021 Jan;397(10270):220-32.

55. Hollander JE, Carr BG. Virtually perfect? Telemedicine for Covid19. N Engl J Med. 2020;382(18):1679-81.

56. Kocoloski A, Ward C, Koontz D, Oddis C, Aggarwal R. Functional measures and patient home self-assessments in the idiopathic inflammatory myopathies [abstract]. Arthritis Rheumatol [Internet]. 2017 [cited 2021 Feb 13];69 (supp 10). Available from: https:// acrabstracts.org/abstract/functional-measures-and-patient-homeself-assessments-in-the-idiopathic-inflammatory-myopathies/

57. Oldroyd A, Yimer B, Little M, Dixon W, Chinoy H. Daily myositis symptom changes collected via a smartphone-based app are associated with flare occurrence - providing evidence of potential digital biomarkers [abstract]. Arthritis Rheumatol [Internet]. 2020 [cited 2021 Feb 14];72 (supp 10). Available from: https://acrabstracts. org/abstract/daily-myositis-symptom-changes-collected-via-asmartphone-based-app-are-associated-with-flare-occurrenceproviding-evidence-of-potential-digital-biomarkers/.
58. Ahmed S, Gupta L. Perception about social media use by rheumatology journals: survey among the attendees of IRACON 2019. Indian J Rheumatol 2020;0(0):0.

59. Lu C-H, Tsang Y-M, Yu C-W, Wu M-Z, Hsu C-Y, Shih TT-F. Rhabdomyolysis: magnetic resonance imaging and computed tomography findings. J Comput Assist Tomogr. 2007 Jun;31(3): 368-74.

60. Delaney-Sathy LO, Fessell DP, Jacobson JA, Hayes CW. Sonography of diabetic muscle infarction with MR imaging, CT, and pathologic correlation. Am J Roentgenol. 2000 Jan;174(1): $165-9$.

61. Khan FY. Rhabdomyolysis: a review of the literature. Neth J Med. 2009 Oct;67(9):272-83.

62. Crum-Cianflone NF. Bacterial, fungal, parasitic, and viral myositis. Clin Microbiol Rev. 2008 Jul;21(3):473-94.

63. Pancheri E, Lanzafame M, Zamò A, Angheben A, Sartoris S, Zorzi A, et al. Benign acute viral myositis in African migrants: a clinical, serological, and pathological study. Muscle Nerve. 2019 Nov;60(5):586-90.

64. Pinal-Fernandez I, Casal-Dominguez M, Mammen AL. Immunemediated necrotizing myopathy. Curr Rheumatol Rep. 2018 Apr;20(4):21.

65. Ritter ML, Pirofski L. Mycophenolate mofetil: effects on cellular immune subsets, infectious complications, and antimicrobial activity. Transpl Infect Dis Off J Transplant Soc. 2009 Aug;11(4):290 7.

Publisher's Note Springer Nature remains neutral with regard to jurisdictional claims in published maps and institutional affiliations. 